\title{
QUELLE PLACE POUR L'AUTONOMIE PROCÉDURALE DES ÉTATS MEMBRES ?
}

\author{
Marjolaine ROCCATI ${ }^{1}$
}

1 La Cour de justice de l'Union européenne et l'autonomie procédurale

1.1 Une autonomie procédurale encadrée

1.2 L'aléa casuistique de cet encadrement

2 Le législateur de l'Union européenne et l'autonomie procédurale

2.1 Une intervention croissante du législateur de l'Union

2.2 Le manque de clarté de l'intervention législative

«Autonomie procédurale », l'expression n'a jamais été autant présente dans les décisions de la Cour de justice de l'Union européenne ${ }^{2}$. Pourtant, si les mots semblent bien connus, la réalité qu'ils recouvrent l'est beaucoup moins.

L'autonomie procédurale des États membres est traditionnellement associée à deux arrêts de la Cour de justice de l'Union européenne de décembre $1976^{3}$, Rewe

1. Maître de conférences, Université Paris Ouest Nanterre La Défense.

2. Pour deux exemples récents, voir CJUE, 17 juillet 2014, Juan Carlos Sánchez. Morcillo, María del Carmen Abril García c. Banco Bilbao Vizcaya Argentaria SA, aff. C-169/14, ECLI:EU:C:2014:2099; CJUE, 5 juin 2014, Bashir Mohamed Ali Mahdi, aff. C-146/14 PPU, ECLI:EU:C:2014:1320.

3. CJCE, 16 décembre 1976, Rewe-Zentralfinanz eG et Rewe-Zentral AG c. Landwirtschaftskammer für das Saarland, aff. 33-76, ECLI:EU:C:1976:188 et Comet BV c. Produktschap voor Siergewassen, aff. 45-76, ECLI:EU:C:1976:191. 
et Comet, dans lesquels la Cour énonce qu'« en l'absence de réglementation communautaire [...], il appartient à l'ordre juridique interne de chaque État membre de désigner les juridictions compétentes et de régler les modalités procédurales des recours en justice destinés à assurer la sauvegarde des droits que les justiciables tirent de l'effet direct du droit communautaire... $»^{4}$.

S'il est ici clairement affirmé, ce renvoi aux modalités procédurales nationales doit être replacé dans un contexte plus général. Il constitue en effet le prolongement naturel d'une jurisprudence plus ancienne. Dès avril 1968, la Cour avait précisé à propos d'une disposition du traité CEE qu'elle « ne limite pas le pouvoir des juridictions nationales compétentes d'appliquer, parmi les divers procédés de l'ordre juridique interne, ceux qui sont appropriés pour sauvegarder les droits substantiels conférés par le droit communautaire $»^{5}$. D'aucuns ont vu, dans cette référence à un choix autonome des procédés par le juge national, l'origine même de l'expression $\mathrm{d}^{\prime}$ « autonomie procédurale $»^{6}$. La Cour ajoutait peu après à propos d'autres dispositions du même traité qu'elles « obligent les autorités et, notamment, les juridictions compétentes des États membres à sauvegarder les intérêts des justiciables affectés par une méconnaissance éventuelle desdites dispositions [...] ; il appartient à l'ordre juridique national de déterminer la juridiction compétente pour assurer cette protection $»^{7}$.

L'autonomie procédurale est associée à l'autonomie institutionnelle des États membres, relative à la désignation des organes compétents pour mettre en œuvre le droit de l'Union. Elle relève de l'autonomie plus générale des États membres dans l'exécution du droit de l'Union, ce dernier s'accomplissant à travers les ordres juridiques nationaux.

Cependant, les autorités européennes se sont toujours montrées réticentes quant à laisser les États membres entièrement libres de leurs choix, veillant à la réalisation du droit de l'Union. Signe de ces réticences, l'expression même d' « autonomie procédurale des États membres » est tardive. Fruit de la doctrine ${ }^{8}$,

4. Arrêt Rewe, point 5 ; arrêt Comet, point 13.

5. CJCE, 4 avril 1968, Firma Gebrüder Lück c. Haupzollamt Köln-Rheinau, aff. 34-67, ECLI:EU:C:1968:24, p. 370 ; CJCE, 3 avril 1968, Firma Molkerei-Zentrale Westfalen/Lippe GmbH c. Hauptzollamt Paderborn, aff. 28-67, ECLI:EU:C:1968:17, p. 228 ; il s'agissait de l'article 95 du Traité CEE sur l'interdiction des impositions intérieures discriminatoires.

6. Cf. D.-U. Galetta : "The expression of "procedural autonomy" - widely referred to in the doctrine, but much less frequent in the language of the ECJ - is probably derived from this expression of "autonomous choice of the means" [de l'arrêt Gebrüder Lück] » [1'expression d'“autonomie procédurale" - à laquelle se réfère largement la doctrine, mais bien moins fréquente dans le langage de la Cour - provient probablement de cette expression de "choix autonome des moyens"], Procedural Autonomy of EU Member States: Paradise Lost?, Berlin, Springer-Verlag, 2011, p. 12.

7. CJCE, 19 décembre 1968, Société par actions Salgoil c. Ministère du Commerce extérieur de la République italienne, aff. 13-68, ECLI:EU:C:1968:54, p. 675 ; il s'agissait des articles 31 et 32 du Traité CEE sur l'interdiction des mesures d'effet équivalent à des restrictions quantitatives.

8. J. Rideau, «Le rôle des États membres dans l'application du droit communautaire », AFDI, 1972, p. 885 ; Droit institutionnel de l'Union et des Communautés européennes, 5 éd., Paris, LGDJ, 2006, p. 896. 
elle semble trouver grâce aux yeux de la Cour de justice davantage dans les conclusions de ses avocats généraux. En 1993, Marco Darmon s'adresse aux magistrats par la phrase d'accroche suivante : «[L]e juge de renvoi vous demande de vous prononcer dans un domaine relevant de ce qu'il est convenu d'appeler l'autonomie procédurale des États membres 9 . » En 1996, Antonio La Pergola cite les arrêts Rewe et Comet de 1976 en invoquant «ce principe d'autonomie procédurale ${ }^{10}$, dans un arrêt relatif aux prestations sociales d'un travailleur migrant ressortissant de l'Union. Au regard de ces deux illustrations, la Cour de justice ne reprend pas ces références. Elle estime dans le premier arrêt que la disposition visée n'est pas d'effet direct ${ }^{11}$. Dans le second, elle impose aux institutions et aux juridictions nationales compétentes en matière de sécurité sociale de respecter les certificats relatifs à l'état des personnes émanant d'autorités compétentes des autres États membres, sans évoquer de renvoi aux procédures nationales dans la détermination des droits aux prestations sociales ${ }^{12}$.

Si l'on revient aux arrêts de 1976, le renvoi effectué par la Cour de justice aux procédures nationales est d'emblée présenté comme conditionnel. L'autonomie procédurale des États membres est une autonomie «en sursis ». Les juges du Kirchberg sont clairs, «en l'absence de réglementation communautaire en la matière, il appartient à l'ordre juridique interne de chaque État membre de désigner les juridictions compétentes [...] ». À l'instar des compétences partagées, les États membres conservent leur compétence en matière procédurale en l'absence d'intervention législative européenne en la matière. La question est donc de savoir si, et le cas échéant quand, le législateur de l'Union est intervenu en la matière, afin de tracer les contours de l'autonomie procédurale des États membres.

Aussi faut-il distinguer, d'une part, la place accordée à l'autonomie procédurale par la Cour de justice de l'Union européenne, suivant l'origine historique de la notion ; d'autre part, la place laissée à cette autonomie procédurale, présentée comme conditionnelle, par le législateur de l’Union (2.).

9. Conclusions de l'avocat général Marco Darmon présentées le 17 novembre 1993, Comitato di coordinamento per la difesa della Cava et autres c. Regione Lombardia et autres, aff. C-236/92, point 41.

10. Conclusions de l'avocat général Antonio La Pergola présentées le 3 décembre 1996, Eftalia Dafeki c. Landesversicherungsanstalt Württemberg, aff. C-336/94, point 5.

11. CJCE, 23 février 1994, Comitato di coordinamento per la difesa della Cava et autres c. Regione Lombardia et autres, aff. C-236/92, ECLI:EU:C:1994:60, point 15, à propos de l'article 4 de la directive 75/442 relative aux déchets.

12. CJCE, 2 décembre 1997, Eftalia Dafeki c. Landesversicherungsanstalt Württemberg, aff. C-336/94, ECLI:EU:C:1997:579, point 21. 


\section{LA COUR DE JUSTICE DE L'UNION EUROPÉENNE ET L'AUTONOMIE PROCÉDURALE}

L'autonomie procédurale des États membres apparaît d'emblée comme encadrée par la Cour de justice de l'Union (1.1), même si cet encadrement intervient de façon aléatoire (1.2).

\subsection{Une autonomie procédurale encadrée}

L'expression n'est plus rare dans la jurisprudence européenne, mais ces références à « l'autonomie procédurale » furent tardives et demeurent parcellaires. Apparaît plus fréquemment la formulation des arrêts Rewe et Comet : le renvoi aux autorités nationales du soin de désigner les juridictions compétentes et de régler les modalités procédurales des recours en justice ${ }^{13}$. Ce renvoi irrigue des domaines toujours plus nombreux et variés du droit de l'Union - droit fiscal, droit de la concurrence, droit de l'environnement, asile et immigration, etc. - dès lors que sont concernés des recours permettant d'assurer la sauvegarde de droits de l'Union. De surcroît, les procédures visées par ces renvois peuvent être de nature administrative et non pas juridictionnelle ${ }^{14}$.

Toutefois, dès les arrêts Rewe et Comet de 1976, la Cour de justice assortit ce renvoi à la procédure nationale de deux limites : les principes d'équivalence et d'effectivité ${ }^{15}$. Suivant le premier, les recours fondés sur le droit de l'Union ne peuvent être moins favorables que ceux similaires de nature interne ; suivant le second, ils ne peuvent rendre pratiquement impossible ou excessivement difficile l'exercice des droits conférés par les normes de l'Union. Presque systématiquement dans sa jurisprudence ultérieure, le renvoi aux procédures nationales est assorti de ces limites, élevées au rang de «principes généraux du droit communautaire ${ }^{16}$.

Équivalence et effectivité sont intimement liées à la notion même d'autonomie procédurale, telle que définie par la Cour de justice dans ses arrêts de 1976. En effet, celle-ci a précisé d'emblée que les recours en justice et leurs modalités procédurales, déterminés en droit national, sont destinés à assurer la sauvegarde

13. Pour un exemple récent, voir CJUE, 23 octobre 2014, Unitrading Ltd. c. Staatssecretaris van Financiën, aff. C-437/13, points 27 et 33, alors même que l'« Autonomie procédurale des États membres » figure dans les mots clefs de la décision.

14. Voir, par exemple, CJCE, 3 février 2000, Charalampos Dounias c. Ypourgio Oikonomikon, aff. C-228/98, ECLI:EU:C:2000:65, point 67, à propos d'une disposition nationale qui prévoit que les contestations relatives à la perception de taxes grevant les produits importés sont résolues dans le cadre d'une procédure administrative.

15. Le principe d'équivalence est posé tel quel dans les arrêts Rewe et Comet ; le principe d'effectivité est précisé dans un arrêt ultérieur : CJCE, 9 novembre 1983, Administration des finances de l'État italien c. SpA San Giorgio, aff. 199/82, ECLI:EU:C:1983:318, point 14.

16. CJCE, 18 septembre 2003, Peter Pflücke c. Bundesanstalt für Arbeit, ECLI:EU:C:2003:477, point 33 . 
des droits que les justiciables tirent du droit de l'Union. Ces droits subjectifs ne peuvent revêtir une importance moindre que leurs homologues de droit national

- principe d'équivalence - et doivent pouvoir être exercés dans l'ordre juridique interne - principe d'effectivité. Équivalence et effectivité sont ainsi les déclinaisons de l'objectif initial, tourné vers la sauvegarde des droits issus des normes européennes. De là dépend la raison d'être du système juridique européen.

Par conséquent, sans même se référer expressément à ces principes, la Cour de justice rappelle aux États membres qu'ils «portent la responsabilité d'assurer, dans chaque cas, une protection effective à ces droits $\gg^{17}$. L'autonomie procédurale est perçue à l'aune de cette protection juridictionnelle effective. À cet égard, il est intéressant de rappeler l'une des occurrences de l'autonomie procédurale, en droit de la concurrence, relative à l'accès d'un justiciable aux documents rédigés par la Commission et envoyés à un tribunal aux fins d'une procédure juridictionnelle particulière ${ }^{18}$. Le Tribunal de première instance avait précisé que «les juridictions tant nationales que communautaires doivent être libres d'appliquer leurs propres règles de procédure en ce qui concerne les pouvoirs du juge, le déroulement de la procédure en général et la confidentialité des pièces du dossier en particulier $»^{19}$. Il s'était ensuite référé à " l'autonomie procédurale des juridictions nationales et communautaires susvisée $\gg^{20}$ et en avait conclu que la décision portant sur l'accès aux informations transmises par la Commission à une juridiction nationale appartenait uniquement à cette dernière sur la base de son droit procédural national ${ }^{21}$. La Cour de justice censure cette décision pour erreur de droit, lui préférant une approche circonstanciée tenant compte de la manière dont s'opère la coopération entre la Commission et les juridictions nationales ${ }^{22}$. Le ton est posé, les règles de procédure nationales doivent être observées, analysées, contrôlées au gré des affaires, pour s'assurer de la façon dont elles peuvent garantir la protection juridictionnelle des droits subjectifs issus des normes européennes.

L'autonomie procédurale des États membres est placée sous le signe de son encadrement, nécessaire à la protection juridictionnelle effective des droits mis en œuvre. Dès lors, s'intéresser à l'autonomie procédurale des États membres requiert

17. CJCE, 9 juillet 1985, Piercarlo Bozzetti c. Invernizzi SpA et ministère du Trésor, aff. 179/84, ECLI:EU:C:1985:306, point 17 ; voir, dès décembre 1968, dans l'arrêt Salgoil, précité : « Les dispositions des articles 31 et 32 obligent les autorités et, notamment, les juridictions compétentes des États membres à sauvegarder les intérêts des justiciables affectés par une méconnaissance éventuelle desdites dispositions en leur assurant une protection directe et immédiate de leurs intérêts... » (p. 675).

18. CJCE, 11 janvier 2000, Royaume des Pays-Bas et Gerard van der Wal c. Commission des Communautés européennes, aff. C-174/98 P et C-189/98 P, ECLI:EU:C:2000:1.

19. TPI, 19 mars 1998, Gerard van der Wal c. Commission des Communautés européennes, aff. T-83/96, Rec. 1998, p. II-00545, point 47.

20. Ibid., point 48 ; érigée par la CJUE au rang de principe, voir point 15.

21. Ibid., point 51 .

22. CJCE, 11 janvier 2000, Royaume des Pays-Bas et Gerard van der Wal..., points 20 à 28. 
d'étudier les contours de cet encadrement. Or une analyse systématique se heurte à l'aléa d'une intervention jurisprudentielle disparate en la matière.

\subsection{L'aléa casuistique de cet encadrement}

Inhérent à sa nature jurisprudentielle, élaboré au gré des arrêts de la Cour de justice de l'Union, l'encadrement de l'autonomie procédurale revêt un caractère ponctuel et casuistique.

Les arrêts Rewe et Comet de 1976 mettent en scène des actions en répétition de l'indu, lorsqu'un État membre a perçu des taxes en violation du droit de l'Union. Dans les deux cas, la Cour a admis la fixation de délais raisonnables de forclusion des recours, laissant présager une autonomie procédurale importante laissée aux États membres. Dans d'autres domaines, la Cour a néanmoins pu se montrer plus incisive. Par exemple, elle a défini les conditions d'octroi de mesures provisoires susceptibles d'être ordonnées par un juge interne à l'encontre d'un acte national exécutant un règlement de l'Union suspecté d'invaliditée ${ }^{23}$. La Cour de justice a aligné ces conditions sur celles des recours formés devant elle ${ }^{24} \mathrm{et}$, par ce biais, les a « communautarisées ». Face à cette ligne de jurisprudence ${ }^{25}$, dès 1996, pouvait-on lire que « les confins [de l'autonomie procédurale] sont vraisemblablement atteints $»^{26}$.

Au regard de l'autonomie procédurale des États membres et de son encadrement par la Cour de justice de l'Union, il est nécessaire de raisonner en termes de lignes de jurisprudence. Par exemple, la Cour de justice a très largement investi le champ des litiges de consommation en ce qui concerne le relevé d'office des clauses abusives ${ }^{27}$, mais quid de ce relevé d'office dans les autres domaines du droit de l'Union? Peut-on le transposer à un cas de discrimination fondée sur le sexe ou à toute autre disposition européenne ? Plus généralement, à qui et dans quelle mesure profite la protection juridictionnelle effective de nature à limiter

23. Le juge national doit édicter de telles mesures provisoires s'il a « des doutes sérieux sur la validité de l'acte communautaire [...] s'il y a urgence et [...] si le requérant est menacé d'un préjudice grave et irréparable, et [...] si cette juridiction prend dûment en compte l'intérêt de la Communauté ».

24. Voir les articles 278 et 279 TFUE.

25. CJCE, 19 juin 1990, The Queen c. Secretary of State for Transport, ex parte: Factortame Ltd. e.a., aff. C-213/89, ECLI:EU:C:1990:257, points 21 et 23 ; CJCE, 21 février 1991, Zuckerfabrik Süderdithmarschen AG c. Hauptzollamt Itzehoe et Zuckerfabrik Soest GmbH c. Hauptzollamt Paderborn, aff. jointes C-143/88 et C-92/89, ECLI:EU:C:1991:65, à propos du sursis à exécution ; CJCE, 9 novembre 1995, Atlanta Fruchthandelsgesellschaft mbH et autres c. Bundesamt für Ernährung und Forstwirtschaft, aff. 465/93, généralisant la jurisprudence précédente à toutes les mesures provisoires.

26. R. Mehdi, « Le droit communautaire et les pouvoirs du juge national de l'urgence (quelques enseignements d'une jurisprudence récente) », RTDE, 1996, n 1 , p. 100.

27. CJUE, 14 mars 2013, Mohamed Aziz c. Caixa d'Estalvis de Catalunya, Tarragona i Manresa (Catalunyacaixa), aff. C-415/11, ECLI:EU:C:2013:164, point 41 et jurisprudence citée. 
l'autonomie procédurale des États membres ? Au sein d'un même domaine, les distinctions jurisprudentielles peuvent être relativement subtiles. En droit de la consommation, la Cour de justice a ainsi censuré une législation espagnole qui ne permettait pas au juge de relever d'office le caractère abusif d'une clause, en l'absence d'opposition du débiteur ${ }^{28}$. À l'inverse, dans un arrêt ultérieur, elle a préservé la législation hongroise qui obligeait le consommateur à avoir recours à une procédure plus coûteuse pour apprécier le caractère abusif d'une clause - sous réserve de validation par le juge de renvoi. En l'espèce, le juge compétent pour se prononcer sur le recours du consommateur visant l'invalidité d'un contrat d'adhésion ne l'était pas pour connaître de la demande dudit consommateur tendant à constater le caractère abusif de clauses contractuelles contenues dans ce même contrat ${ }^{29}$. Entre protection juridictionnelle du consommateur et autonomie procédurale des États membres, la balance de la justice oscille sans être toujours bien prévisible.

Face à cette situation, les éclaircissements quant à l'encadrement de l'autonomie procédurale des États membres ne semblent pouvoir être attendus du législateur de l’Union.

\section{LE LÉGISLATEUR DE L'UNION EUROPÉENNE ET L'AUTONOMIE PROCÉDURALE}

L'autonomie procédurale des États membres, encadrée par la Cour de justice, s'est réduite de par l'intervention du législateur dans le domaine procédural (2.1). Encore discrète, cette intervention se place dans la continuité de la jurisprudence et manque de clarté (2.2).

\subsection{Une intervention croissante du législateur de l'Union}

Le législateur de l'Union s'immisce dans la procédure des États membres, pour assurer la protection juridictionnelle des droits contenus dans nombre d'instruments

28. CJUE, 14 juin 2012, Banco Español de Crédito SA c. Joaquin Calderón Camino, aff. C-618/10, ECLI:EU:C:2012:349, point 57 : « La directive 93/13 doit être interprétée en ce sens qu'elle s'oppose à une réglementation d'un État membre [...] qui ne permet pas au juge saisi d'une demande d'injonction de payer d'apprécier d'office, in limine litis ni à aucun autre moment de la procédure, alors même qu'il dispose des éléments de droit et de fait nécessaires à cet effet, le caractère abusif d'une clause [...], en l'absence d'opposition formée par ce dernier ».

29. CJUE, 12 février 2015, Nóra Baczó et János István Vizsnyiczai c. Raiffeisen Bank Zrt, aff. C-567/13, point 59: «L'article 7, paragraphe 1, de la directive 93/13 [...] ne s'oppose pas à une règle de procédure nationale en vertu de laquelle une juridiction locale compétente pour se prononcer sur le recours d'un consommateur visant l'invalidité d'un contrat d'adhésion ne l'est pas pour connaître de la demande dudit consommateur tendant à constater le caractère abusif de clauses contractuelles contenues dans ce même contrat, sauf s'il s'avérait que le dessaisissement de la juridiction locale entraîne des inconvénients procéduraux de nature à rendre excessivement difficile l'exercice des droits qui sont conférés au consommateur par l'ordre juridique de l'Union. Il appartient à la juridiction nationale de procéder aux vérifications nécessaires à cet égard. » 
de droit dérivé. Ce droit à une protection juridictionnelle effective est aujourd'hui bien en vue en droit de l'Union, affirmé à l'article 19 \$ 1 du Traité sur l'Union européenne ${ }^{30}$, complété par l'article 47 de la Charte des droits fondamentaux qui garantit le « droit à un recours effectif et à accéder à un tribunal impartial $»^{31}$ lorsqu'est mis en œuvre le droit de l'Union.

La portée de la protection juridictionnelle mentionnée demeure imprécise. Dans l'article 52 de la Charte, il est prévu pour les droits correspondant à des droits garantis par la Cour européenne des droits de l'homme que « leur sens et leur portée sont les mêmes que ceux que leur confère ladite convention ». La Cour de justice a ainsi admis que l'article 47, deuxième alinéa, de la Charte des droits fondamentaux correspondait à l'article $6 \S 1$ de la $\mathrm{CEDH}^{32}$. Au-delà de ces indications, ces droits ne sont pas précisés dans le cadre des instruments de l’Union qui s'immiscent dans la procédure des États membres.

En effet, avant même l'affirmation nette d'un droit à une protection juridictionnelle effective, le législateur de l'Union en avait tracé certains contours au gré d'instruments de droit dérivé. Une directive, adoptée en 1996, permet au travailleur détaché d'intenter une action en justice dans l'État membre sur le territoire duquel il est détaché, afin de faire valoir les droits qu'il tire de l'application de la directive $^{33}$. Dans le cadre de la législation européenne relative à la publicité trompeuse et à la publicité comparative, la possibilité d'actions collectives est introduite ${ }^{34}$. En droit de la concurrence, les règles européennes répartissent la charge de la preuve entre la partie ou l'autorité qui allègue une entente et l'entreprise ou l'association d'entreprises qui se prévaut d'une exemption ${ }^{35}$. Ces illustrations, non exhaustives, sont révélatrices de l'intervention européenne dans la procédure des États membres au gré de divers instruments de droit dérivé.

30. JO C 326, 26 octobre 2012, pp. 13-390 : « [1]es États membres établissent les voies de recours nécessaires pour assurer une protection juridictionnelle effective dans les domaines couverts par le droit de l'Union ».

31. JO C 326, 26 octobre 2012, pp. 391-407 : « Toute personne dont les droits et libertés garantis par le droit de l'Union ont été violés a droit à un recours effectif devant un tribunal dans le respect des conditions prévues au présent article.

Toute personne a droit à ce que sa cause soit entendue équitablement, publiquement et dans un délai raisonnable par un tribunal indépendant et impartial, établi préalablement par la loi. Toute personne a la possibilité de se faire conseiller, défendre et représenter.

Une aide juridictionnelle est accordée à ceux qui ne disposent pas de ressources suffisantes, dans la mesure où cette aide serait nécessaire pour assurer l'effectivité de l'accès à la justice. »

32. CJUE, 22 décembre 2010, DEB Deutsche Energiehandels- und Beratungsgesellschaft mbH c. Bundesrepublik Deutschland, aff. C-279/09, ECLI:EU:C:2010:811, point 32.

33. Directive 96/71/CE du 16 décembre 1996 concernant le détachement de travailleurs effectué dans le cadre d'une prestation de services, JO L 018, 21 janvier 1997, pp. 1-6, article 6.

34. Directive 84/450/CEE, article 4, paragraphe 1 , repris à l'article 5, paragraphe 1 de la directive 2006/114/CE ; voir également l'article 23 de la directive 2011/83/UE du 25 octobre 2011 relative aux droits des consommateurs, modifiant la directive 93/13/CEE du Conseil et la directive 1999/44/ CE du Parlement européen et du Conseil et abrogeant la directive 85/577/CEE du Conseil et la directive 97/7/CE du Parlement européen et du Conseil, JO L 304, 22 novembre 2011, pp. 64-88.

35. Règlement $(\mathrm{CE}) \mathrm{n}^{\circ} 1 / 2003$ du 16 décembre 2002 relatif à la mise en œuvre des règles de concurrence prévues aux articles 81 et 82 du Traité, $J O$ L 1, 4 janvier 2003, pp. 1-25, article 2. 
Aux côtés de ces interventions législatives ponctuelles, des instruments entiers de droit dérivé ont pu investir le champ de la procédure des États membres. Récemment a été adoptée la directive du 26 novembre 2014 relative à certaines règles régissant les actions en dommages et intérêts en droit national pour les infractions aux dispositions du droit de la concurrence ${ }^{36}$. Parallèlement, une directive du 21 mai 2013 a mis en place un système de règlement extrajudiciaire des litiges de consommation ${ }^{37}$. Elle est associée à un règlement permettant la résolution en ligne des litiges de consommation ${ }^{38}$. Les règles ainsi élaborées par le législateur de l'Union dans le domaine procédural peuvent simplement mentionner la nécessité d'un recours, ou être beaucoup plus précises, détaillant par exemple les pouvoirs des juridictions en matière de production de preuves dans la directive régissant les actions en dommages et intérêts en droit de la concurrence. Elles sont de plus en plus nombreuses en raison du champ toujours plus large d'intervention du législateur de l'Union. Cette immixtion croissante, y compris donc dans le domaine procédural, manque de clarté.

\subsection{Le manque de clarté de l'intervention législative}

Le législateur de l'Union investit le champ de la procédure des États membres, sans chercher toutefois à se démarquer d'une autonomie procédurale préexistante des États membres. La référence à l'expression même d'autonomie procédurale se retrouve de façon marginale uniquement dans l'un des considérants de deux instruments législatifs du droit de l'Union, une décision-cadre de 2009 relative à la prévention et au règlement des conflits en matière d'exercice de la compétence dans le cadre des procédures pénales ${ }^{39}$ et une directive de 2007 relative aux procédures de recours en matière de passation des marchés publics ${ }^{40}$.

Sans se référer expressément à l'autonomie procédurale des États membres, le législateur de l'Union rappelle parfois le renvoi aux procédures nationales tout en mettant l'accent sur ses limites. Ainsi, la directive du 26 novembre 2014, relative

36. Directive 2014/104/UE du 26 novembre 2014 relative à certaines règles régissant les actions en dommages et intérêts en droit national pour les infractions aux dispositions du droit de la concurrence des États membres et de l'Union européenne, JO L 349, 5 décembre 2014, pp. 1-19.

37. Directive 2013/11/UE du 21 mai 2013 relative au règlement extrajudiciaire des litiges de consommation et modifiant le règlement (CE) $\mathrm{n}^{\circ}$ 2006/2004 et la directive 2009/22/CE (directive relative au RELC), JO L 165, 18 juin 2013, pp. 63-79.

38. Règlement (UE) $n^{\circ}$ 524/2013 du 21 mai 2013 relatif au règlement en ligne des litiges de consommation et modifiant le règlement $(\mathrm{CE}) \mathrm{n}^{\circ}$ 2006/2004 et la directive 2009/22/CE (règlement relatif au RLLC), JO L 165, 18 juin 2013, pp. 1-12.

39. Décision-cadre 2009/948/JAI du 30 novembre 2009 relative à la prévention et au règlement des conflits en matière d'exercice de la compétence dans le cadre des procédures pénales, $J O$ L 328 , 15 décembre 2009, pp. 42-47, 8 considérant.

40. Directive 2007/66/CE du 11 décembre 2007 modifiant les directives 89/665/CEE et 92/13/CEE du Conseil en ce qui concerne l'amélioration de l'efficacité des procédures de recours en matière de passation des marchés publics, $J O$ L 335, 20 décembre 2007, pp. 31-46, 34e considérant. 
à certaines règles régissant les actions en dommages et intérêts en droit national pour les infractions aux dispositions du droit de la concurrence, met en avant les principes d'effectivité et d'équivalence ${ }^{41}$. Par ailleurs, l'objectif est posé dès le premier article : établir des règles garantissant une protection équivalente, dans toute l'Union, à toute personne ayant subi un préjudice causé par une infraction au droit de la concurrence.

Cet instrument récent paraît illustrer la perception du législateur de l'Union de l'autonomie procédurale des États membres. En réalité, il n'y a pas d'autonomie procédurale, car il n'y a pas d'autonomie de la procédure. Celle-ci est uniquement perçue dans sa nature servante, à travers les droits substantiels qu'elle permet de garantir. Si ceux-ci ne sont pas suffisamment garantis par l'application de procédures nationales, la Cour de justice intervient pour encadrer ses dernières, suivie du législateur qui recherche une protection équivalente au sein de l'Union européenne.

Faut-il dès lors faire de la procédure une discipline autonome au sein de l'Union européenne ? Pourraient ainsi être clarifiées des notions qui se recoupent et dont l'articulation n'est pas toujours aisée : quels liens entre la «protection juridictionnelle effective » de l'article $19 \S 1$ du Traité sur l'Union européenne, le « droit à un recours effectif » de l'article 47 de la Charte et le «principe d'effectivité minimale » des arrêts Rewe et Comet de 1976 qui encadre tout renvoi aux procédures nationales ? Quel contenu pour ces notions ? Souvent présentes comme des coquilles vides dans les instruments du droit de l'Union, la Cour de justice a alors la charge d'en définir le contenu, éventuellement en se reposant sur le juge national.

Les résistances des États membres sont sans doute encore trop fortes pour que le législateur de l'Union investisse davantage le champ de la procédure, vingt ans après l'échec de la commission Storme, chargée de rédiger un Code judiciaire européen $^{42}$. Par ailleurs, l'heure ne paraît pas favorable à un renforcement de l'autonomie de la procédure ; les États membres paraissent au contraire suivre la vision européenne. C'est ainsi, par exemple, en France que les actions en justice des associations, dont la récente action de groupe introduite en mars 2014, figurent dans le Code de la consommation ${ }^{43}$ et non dans le Code de procédure civile.

Si l'heure ne semble pas à une autonomie plus grande de la procédure, peutêtre est-il en revanche nécessaire de tenter une plus grande systématisation de cet encadrement procédural européen, en insérant par exemple la jurisprudence

41. Voir considérants (11), (37), (46) ainsi que l'article 4 de la directive 2014/104/UE : il s'agit d'assurer l'effectivité du droit, conféré par l'Union, à réparation intégrale du préjudice causé par une infraction au droit de la concurrence et l'équivalence entre actions en dommages et intérêts découlant d'infractions à l'article 101 ou 102 TFUE et celles découlant d'infractions au droit national.

42. M. Storme (dir.), Rapprochement du droit judiciaire de l'Union européenne - Approximation of Judiciary Law in the European Union, Dordrecht, Martinus Nijhoff, 1994.

43. Livre IV : Les associations de consommateurs, Titre II : Actions en justice des associations, Chapitre III : Action de groupe. 
européenne dans les instruments pertinents, lors de la refonte de ces derniers. Au contraire, alors même que la Cour de justice avait déjà bien affirmé sa jurisprudence relative au relevé d'office des clauses abusives, le législateur européen s'est montré très discret dans sa proposition de directive de 2008 et s'est contenté d'une vague référence dans l'un des considérants : «Les États membres doivent s'assurer que leurs juridictions ou leurs autorités administratives disposent de moyens adéquats et efficaces pour empêcher que l'application de clauses abusives dans les contrats conclus avec les consommateurs persiste ${ }^{44}$.»

Pour davantage de clarté, peut-être faudrait-il commencer par ne plus se référer à « l'autonomie procédurale » et lui préférer l'expression de « compétence procédurale fonctionnelle $\gg^{45}$ en droit de l'Union. Et travailler à éviter le schéma trop souvent rencontré en la matière : le législateur de l'Union laisse tout éclaircissement aux soins de la Cour de justice, qui se repose à son tour sur le juge national. Pour plus de cohérence juridique, peut-être serait-il temps d'inverser la séquence.

44. Proposition de directive du Parlement européen et du Conseil relative aux droits des consommateurs $\{$ SEC(2008) 2544\} \{SEC(2008) 2545\} \{SEC(2008) 2547\}, point 55.

45. D.-U. Galetta, Procedural Autonomy of EU Member States: Paradise Lost?, op. cit., p. 123. 
